\title{
USO DO COPINHO NO ALOJAMENTO CANGURU
}

\author{
Cup-Feeding in Kangaroo Mother Care
}

\author{
Vívian Passos Lima ${ }^{(1)}$, Adriana de Medeiros Melo ${ }^{(2)}$
}

\section{RESUMO}

Objetivo: avaliar o manejo das mães no uso do copinho e analisar os aspectos que interferem para administração dessa técnica. Métodos: a amostra foi composta de 30 binômios mãe/bebê. O estudo desenvolvido foi transversal de correlação. Foram realizadas coletas nos prontuários das mães e do bebê, executada uma observação das mães ofertando a dieta no copinho e aplicado um questionário com perguntas sobre o uso do copo. A análise dos resultados foi realizada por meio do programa software estatístico, SPSS 13.0. Aplicou-se testes de estatística descritiva, mais especificamente a distribuição das freqüências e das percentagens relativas de cada dado observado. Em relação à associação entre as variáveis estudadas foram utilizados os seguintes testes: teste Qui-Quadrado, com nível de significância de $5 \%(p \leq 0,05)$ e o teste de Correlação Bivariada com o grau de correlacionamento linear de Spearman $(R)$, utilizando o nível de significância de $5 \%(p \leq 0,05)$. Resultados: há relação significante da postura do bebê, posição do copo, volume administrado e orientação dos profissionais de saúde às mães sobre o uso do copinho. Conclusão: o copinho pode ser manejado pelas mães, mas estas necessitam de orientações sobre o uso da técnica pelos profissionais de saúde; principalmente em relação ao cuidado com a posição do copo e volume de leite.

DESCRITORES: Leite Humano; Métodos de Alimentação; Recém-Nascido; Prematuro

\section{INTRODUÇÃO}

A política de saúde materno-infantil no Brasil tem priorizado ações de promoção, proteção e apoio ao aleitamento materno (AM), por ser uma estratégia fundamental para a redução da mortalidade infantil e melhoria da qualidade de saúde da população ${ }^{1}$. No entanto, as taxas de prevalência estão muito longe de atingir as recomendações da Organização Mundial da Saúde (OMS), que preconiza o aleitamento materno exclusivo (AME) por seis meses e da manutenção da amamentação após a introdução de outros alimentos até os dois anos de vida ou mais ${ }^{2}$.

Uma forma bem eficiente para estimular a amamentação natural é através do Método Mãe Canguru, definido pelo Ministério da Saúde como sendo

(1) Fonoaudióloga; Otorhinus - Centro de Fonoaudiologia, otorrinolaringologia, patologias alérgicas e respiratórias; Centro Auditivo Widex; Especializanda em Audiologia Clínica pelo Instituto Alfa Unidade II.

(2) Fonoaudióloga; Professora Auxiliar da Disciplina Abordagem Terapêutica em Motricidade Oral do Departamento de Fonoaudiologia da Universidade Estadual de Ciências da Saúde de Alagoas; Mestranda em Saúde da Criança e do Adolescente pela Universidade Federal de Pernambuco. uma assistência neonatal de contato pele a pele precoce entre o recém-nascido $(\mathrm{RN})$ de baixo peso prematuro e sua mãe, permitindo maior participação dos pais no cuidado com seu filho ${ }^{3}$; além de proporcionar redução da morbidade e mortalidade infantis; benefícios psicoafetivos e neurossensoriais, relacionados à prática do $\mathrm{AM}$ e respostas fisiológicas mais satisfatórias ${ }^{3-6}$. Este método é desenvolvido em três etapas: a primeira em unidades de terapia intensiva neonatal ou de cuidados intermediários; a segunda no alojamento canguru; e a terceira após a alta hospitalar, nos ambulatórios de seguimento ${ }^{4,5}$.

O AME configura-se quando a criança recebe somente leite materno, diretamente da mama ou extraído desta, e nenhum outro líquido ou sólido, com exceção de gotas ou xaropes de vitaminas, minerais e/ou medicamentos quando necessários ${ }^{7}$. A amamentação exclusiva é especialmente indicada para recém-nascidos (RNs) de baixo peso ou peso insuficiente, já que estes necessitam de maior aporte energético e biodisponibilidade de nutrientes para recuperação do estado nutricional e proteção contra possíveis infecções ${ }^{8}$. Dentre os inúmeros benefícios comprovados cientificamente, a amamentação fortalece o vínculo mãe-bebê, fornece valores nutricionais indiscutíveis, propicia o 
aumento de anticorpos e ganho de peso, além de proporcionar a harmonia do sistema estomatognático ${ }^{9}$.

Tal fato é explicado visto que durante a amamentação o bebê realiza uma ampla abertura mandibular para que ocorra um vedamento labial adequado, em que a parte anterior dos lábios com eversão e a língua se apóia na gengiva inferior, curvando-se para cima. Dessa forma, ocorre a pressão negativa formada por movimentos da mandíbula associados aos movimentos dos lábios, bochechas e coxins de gordura. A mandíbula realiza um ciclo de movimentos: o abaixamento para a abertura da boca, protrusão mandibular, elevação e compressão dos seios lactíferos e a retrusão ${ }^{10}$.

Entretanto, em algumas situações, o AME não pode ser realizado, como nos casos de crianças que necessitam de suplementação; sendo necessário administrar a dieta com um método alternativo ${ }^{11}$. A mamadeira é o método mais comum de suplementação, porém evidências científicas mostram que seu uso interfere significativamente na amamentação, pois os bicos de mamadeira podem ter impacto negativo no desenvolvimento do comportamento de sucção do lactente ${ }^{12}$. A exposição ao uso de bicos artificiais diminui o tempo de aleitamento materno e do contato mãe-bebê, além de favorecer o aparecimento de diversas patologias gerais de saúde e fonoaudiológicas ${ }^{13}$.

Diante disso, o copinho vem se tornando um método freqüente de alimentação de RNs nos berçários incentivada pela Iniciativa Hospital Amigo da Criança ${ }^{14}$ por ser uma técnica alternativa de alimentação, utilizada inclusive em alguns casos de fracasso inicial com o aleitamento no peito, tal como acontece em alguns RNs que não coordenam os reflexos de sucção e deglutição com a respiração nos primeiros dias de vida. O principal objetivo desta técnica é promover um método artificial de alimentação seguro para os recém-nascidos pré-termo (RNPTs) de baixo peso, até que fiquem aptos o suficiente para realizarem a amamentação exclusiva no peito ${ }^{15}$.

O procedimento de administração do leite com o uso do copinho deve ser realizado da seguinte maneira: a mãe/cuidador deve segurar o bebê em estado de alerta, envolvendo-o em um lençol para que o leite não seja derramado pela movimentação de seus membros superiores. O RN deve estar sentado ou semi-sentado no colo da mãe ou do cuidador. Posteriormente, deve-se encostar a borda do copinho no lábio inferior do bebê, inclinando-o até que o leite toque o seu lábio inferior. Aguardar que o bebê retire o leite, sorvendo-o; e em seguida o degluta. Não é necessário derramar o leite na boca do RN ${ }^{16,17}$.
As vantagens da alimentação no copinho são: o bebê determina seu próprio consumo, referente ao tempo e a quantidade; dispende pouca energia; estimula o desenvolvimento e a coordenação dos reflexos de sucção e deglutição; estimula a secreção da saliva e das lipases da língua, tornando a digestão do leite materno mais eficiente e é um método fácil de oferta da dieta ${ }^{15}$. Os movimentos de língua e da mandíbula realizados durante o uso do copinho são similares aos movimentos necessários para o sucesso da amamentação, e seu uso desenvolve os músculos responsáveis por esses movimentos, além de promover uma experiência oral positiva reduzindo o tempo de uso de sonda orogástrica (SOG) e facilitar o desenvolvimento da relação entre o prematuro e sua mãe e/ou pai ${ }^{18}$. As desvantagens são: o bebê costuma babar; pode ser que pela facilidade substitua a amamentação natural; pode haver formação de bolhas no leite ${ }^{15}$ e o cuidador pode despejar o leite diretamente na boca da criança, ficando esta susceptível a engasgos e aspirações ${ }^{18}$.

Durante a alimentação por copinho, os RNPTs apresentam inicialmente um movimento de lambida, observando a protrusão da língua para obter o leite, ação vital para o sucesso da amamentação, visto que a habilidade de distensão da língua é fundamental para a extração do leite dos ductos mamilares. Posteriormente, este movimento vai sendo substituído pelo movimento de sorver ${ }^{15}$. Enquanto se alimentam com copinho, os RNPTs podem controlar os movimentos de sorver, a respiração é mais fácil de ser coordenada e a deglutição ocorre quando o recém-nascido estiver apto para realizar esta função ${ }^{19}$.

A ação de sorver é o movimento predominante realizado pelos RNPTs para obtenção do leite. Não ocorre a protrusão da língua durante essa ação, pois a cavidade oral encontra-se parcialmente fechada, podendo ocorrer mecanismo parecido com o denominado "confusão de bicos" ${ }^{20}$.

Há grande controvérsia na literatura quanto à eficácia do uso do copinho como método alternativo de alimentação, principalmente no que se refere ao mecanismo de "confusão de bicos", conceituado como uma dificuldade dos neonatos em exibir uma configuração oral correta, em dominar a técnica e o padrão de sucção necessário para o sucesso da amamentação depois da exposição a mamadeiras e outros bicos artificiais ${ }^{14,21}$. Porém, pode-se observar que a técnica do copinho é proveitosa e efetiva e que permite uma posterior amamentação ao peito bem sucedida sem que ocorra a confusão de bicos ${ }^{22}$.

O aleitamento materno continua sendo o método de alimentação mais adequado para lac- 
tentes, porém o uso do copo é recomendável nos momentos de impossibilidade da amamentação, pois, em estudo com o recurso da eletromiografia de superfície, a musculatura ativa em ambos os métodos (masseter, temporal e bucinador) é a mesma com a vantagem de não provocar a "confusão de bicos" ${ }^{23}$.

Apesar dos inúmeros benefícios do AM, os indicadores do Sistema de Informação da Atenção Básica (SIAB) revelam que a proporção do AME no ano de 2003, até os 04 meses de idade, no Brasil é de $62,3 \%$; na região nordeste de $57,5 \%$ e no Estado de Alagoas de $49,9 \%{ }^{24}$.

Para a saúde coletiva as principais situações que facilitam o desmame precoce são a utilização da mamadeira juntamente com as fórmulas de leite artificiais ${ }^{17}$. Há outros fatores também relacionados ao desmame precoce: grau de escolaridade da mãe, idade e trabalho maternos, urbanização, condições de parto, incentivo do cônjuge e de parentes ${ }^{25}$.

Diante do baixo índice de AME até os 6 meses no Estado de Alagoas registrado pela OMS em 2003 de $49,9 \%$, este trabalho tem como objetivo avaliar o manejo das mães com o uso do copinho e analisar os aspectos que interferem para a administração adequada dessa técnica.

\section{MÉTODOS}

A pesquisa foi desenvolvida na Enfermaria Canguru da Maternidade Escola Santa Mônica (MESM) em Maceió-AL, que é uma Instituição de referência para todo o Estado, visto que atende gestantes de alto risco pelo Sistema Único de Saúde (SUS) e é a única maternidade a possuir a referida enfermaria. A coleta de dados foi realizada no período de dezembro de 2005 a maio de 2006.

O estudo envolveu 30 binômios mãe/bebê de RNPTs de baixo peso, com o tipo de estudo transversal de correlação. Foram realizadas entrevistas com as mães que se adequavam aos seguintes critérios de inclusão: mães de RNPTs de baixo peso, admitidas no Alojamento Canguru da MESM, os quais estavam recebendo dieta via oral com uso do complemento no copinho.

A pesquisa foi explicada verbalmente às genitoras e o Termo de Consentimento Livre e Esclarecido foi entregue e assinado. Em seguida, foi executada a coleta de dados nos prontuários da mãe e do recém-nascido, no que se refere à idade e escolaridade das mães, número de gestação anterior, idade gestacional corrigida do bebê, e volume da dieta administrado no dia da coleta.

No momento em que as mães estavam oferecendo a dieta via oral através do copinho para o $\mathrm{RN}$ na enfermaria canguru, as pesquisadoras rea- lizaram uma observação da postura da mãe, postura do bebê e da posição do copo, analisando se esses critérios encontravam-se adequados ou inadequados, ou seja, durante a alimentação com o copinho, seria necessária que a posição do copo estivesse levemente inclinada sobre o lábio inferior com o bebê delimitando seu próprio consumo, a mãe deveria estar com uma postura ereta e com apoio nos membros inferiores e o bebê sentado ou semi-sentado no colo da dela. Além de observar ritmo/velocidade, pausas e escape prematuro de leite durante a alimentação do RN.

Após a oferta da dieta, foi aplicado um questionário às mães, constituído por perguntas sobre orientações dos profissionais da saúde, conhecimento quanto à técnica do copinho e tempo de administração desta pela mãe, para que fossem analisadas as percepções que elas possuem sobre o uso do copinho, assim como a importância deste para seu bebê.

Este Projeto foi analisado e aprovado pelo Comitê de Ética em Pesquisa da Universidade Estadual de Ciências da Saúde de Alagoas (UNCISAL), no dia 27 de outubro de 2005, com o protocolo número 458 , e pela comissão de ética do núcleo de ensino e pesquisa da MESM.

A análise dos resultados foi realizada por meio do programa software estatístico, SPSS 13.0 (Statistical Package for the Social Sciences). Aplicou-se testes de estatística descritiva, mais especificamente a distribuição das freqüências e das percentagens relativas de cada dado observado. Em relação à associação entre as variáveis estudadas foram utilizados os seguintes testes: teste Qui-Quadrado, com nível de significância de $5 \%(p \leq 0,05)$ e o teste de Correlação Bivariada com o grau de correlacionamento linear de Spearman (R), utilizando o nível de significância de $5 \%(p \leq 0,05)$.

\section{RESULTADOS}

A primeira variável analisada foi a idade das mães. Observou-se que $30 \%$ das mães possuem idade entre 15 e 19 anos, 36,6\% encontram-se entre 20 a 29 anos e 33,3\% estão na faixa etária entre 30 e 39 anos de idade. Quanto ao nível de escolaridade das mães, apenas 3,3\% completaram o ensino superior, $6,6 \%$ possuem o ensino superior incompleto, $26,6 \%$ apresentam o ensino médio completo, $46,6 \%$ possuem o ensino fundamental incompleto e 10\% não são alfabetizadas.

Metade das mães, $50 \%$ teve gestação anterior, sendo $26,6 \%$ apenas uma e $23,3 \%$ mais de uma gestação. No que se refere ao tempo de administração, em dias, da dieta no copinho pela mãe, mais da metade da amostra, $80 \%$, administram a dieta 
entre 1 e 5 dias e apenas $20 \%$ entre 6 a 11 dias. A disposição da idade gestacional (IG) corrigida do RN foi: $20 \%$ estavam entre 33 e 35 semanas, $46,6 \%$ entre 36 e 37 semanas e $33,3 \%$ encontraram-se com mais de 38 semanas.

Foi estudada também a orientação fornecida pelos profissionais da saúde às mães sobre a importância e a forma de usar o copinho durante a oferta da dieta, em que $63,3 \%$ das mães receberam orientação e 33,3\% não receberam. Quanto ao conhecimento das mães acerca da importância do uso do copinho pode-se demonstrar que $40 \%$ possuem algum conhecimento enquanto que $60 \%$ não apresentam conhecimento.

O volume de leite ofertado no copinho durante a administração das dietas foi de $15 \mathrm{ml}$ em 33,3\%, entre 20 e $25 \mathrm{ml}$ em $20 \%$ e de $30 \mathrm{ml}$ em 46,6\%. Durante a oferta da dieta no copinho, $53,3 \%$ dos RNs apresentaram-se ritmados para sorver o leite; $50 \%$ tiveram pausas longas; e 43,3\% apresentaram escape prematuro de leite. Não foram observados episódios de tosse e engasgo na amostra do estudo.

Tabela 1 - Características descritivas das mães e dos bebês durante o período de alimentação no copinho, Maceió, 2006

\begin{tabular}{ccc}
\hline Variáveis & Média & Desvio Padrão \\
\hline Idade da Mãe & 25,3 & 7,2 \\
Idade Gestacional & 36,9 & 2,7 \\
$\quad$ Corrigida & & 2,5 \\
$\begin{array}{c}\text { Tempo de } \\
\text { administração da }\end{array}$ & 3,6 & \\
dieta no copinho & & 6,7 \\
$\begin{array}{c}\text { Volume de leite } \\
\text { administrado }\end{array}$ & 23,8 & \\
\hline
\end{tabular}

A Tabela 1 ilustra as medidas descritivas das variáveis: idade da mãe, IG corrigida do bebê, tempo de alimentação no copinho e volume de leite. As Tabelas 2 e 3 demonstram os resultados do teste Qui-Quadrado. Não foi observada relação estatisticamente significante na postura da mãe e posição do copo, porém obteve-se esta relação quanto à postura do bebê, em que no presente estudo apresentou-se adequada. No que se referem ao ritmo/velocidade do $\mathrm{RN}$, pausas longas $\mathrm{e}$ escape prematuro de leite, não foram observadas relações estatisticamente significantes.

A aplicação do teste de correlação bivariada utilizando o coeficiente linear de Spearman apresentou os seguintes resultados:

Não foi observada relação estatisticamente significante do uso do copinho e as variáveis idade e escolaridade das mães, presença de gestação anterior, tempo de administração da dieta no copinho pelas mães e IG corrigida. As análises estão expostas nas Tabelas 4 e 5. A importância da orientação fornecida pelos profissionais da saúde para o sucesso da administração da dieta no copinho também está ilustrada na Tabela 4, apresentando relação estatisticamente significante. O mesmo não foi evidenciado com o conhecimento das mães sobre a importância do uso do copinho para a administração adequada dessa técnica.

A posição do copo realizada pelas mães apresentou relação estatisticamente significante quando relacionada com os aspectos de alimentação do RN. O volume de leite ofertado na dieta do RN merece ser mencionado, pois, nesse estudo, observou-se que quanto maior o volume, maiores chances de ocorrerem pausas longas e escape prematuro de leite, relação esta estatisticamente significante. Esses dados podem ser observados na Tabela 5.

Tabela 2 - Comparação do manejo do copinho (adequado e inadequado), relacionado à postura da mãe, do bebê e posição do copo, Maceió, 2006

\begin{tabular}{cccc}
\hline & Postura da Mãe & Postura do Bebê & Posição do Copo \\
\hline $\begin{array}{c}\text { Teste Qui-Quadrado } \\
\text { (Valores de P) }\end{array}$ & 0,06 & $0,00^{*}$ & 0,06 \\
\hline
\end{tabular}

Teste qui-quadrado * $=\mathrm{P}<0,05$ (Significativo)

Tabela 3 - Comparação do comportamento da alimentação do bebê (presença e ausência das variáveis abaixo) durante a oferta da dieta no copinho, Maceió, 2006

\begin{tabular}{cccc}
\hline & $\begin{array}{c}\text { Ritmo/Velocidade do RN } \\
\text { em Sorver o Leite }\end{array}$ & $\begin{array}{c}\text { Pausas Longas } \\
\text { para Sorver o Leite }\end{array}$ & $\begin{array}{c}\text { Escape Prematuro } \\
\text { de Leite }\end{array}$ \\
\hline $\begin{array}{c}\text { Teste Qui-Quadrado } \\
\text { (Valores de P) }\end{array}$ & 0,71 & 1,00 & 0,46 \\
\hline
\end{tabular}

Teste qui-quadrado: * $=\mathrm{P}<0,05$ (Significativo) 
Tabela 4 - Características maternas, modo e tempo de administração da dieta no copinho, Maceió, 2006

\begin{tabular}{|c|c|c|c|}
\hline Variáveis & Postura da Mãe & Postura do Bebê & Posição do Copo \\
\hline Idade da Mãe & 0,87 & 0,06 & 0,75 \\
\hline Escolaridade da Mãe & 0,25 & 0,18 & 0,57 \\
\hline Gestação Anterior & 0,48 & 0,30 & 0,21 \\
\hline $\begin{array}{l}\text { Tempo que a Mãe Administra a } \\
\text { Dieta no Copinho }\end{array}$ & 0,63 & 0,58 & 0,33 \\
\hline $\begin{array}{l}\text { Orientação pelos profissionais de } \\
\text { saúde sobre como usar o copinho }\end{array}$ & 0,06 & 0,69 & $0,00^{*}$ \\
\hline $\begin{array}{l}\text { Conhecimento das mães sobre a } \\
\text { importância do uso do copinho }\end{array}$ & 0,44 & 0,24 & 0,12 \\
\hline
\end{tabular}

Correlação Bivariada * $=p<0,05$ (Significativo)

Tabela 5 - Características apresentadas pelo bebê durante a oferta da dieta com o uso do copinho relacionadas ao comportamento de alimentação do bebê (presença e ausência das variáveis abaixo), Maceió, 2006

\begin{tabular}{cccc}
\hline Variáveis & $\begin{array}{c}\text { Ritmo/Velocidade do RN } \\
\text { em Sorver o Leite }\end{array}$ & $\begin{array}{c}\text { Pausas Longas } \\
\text { para Sorver o Leite }\end{array}$ & \multicolumn{2}{c}{$\begin{array}{c}\text { Escape Prematuro de } \\
\text { Leite }\end{array}$} \\
\hline IG Corrigida & 0,24 & 0,28 & 0,47 \\
Volume & 0,09 & $0,04^{*}$ & $0,02^{*}$ \\
Posição do Copo & $0,00^{*}$ & $0,00^{*}$ & $0,00^{*}$ \\
\hline
\end{tabular}

Correlação Bivariada * $=p<0,05$ (Significativo)

\section{DISCUSSÃO}

Não foi observada relação estatisticamente significante $(p>0,05)$ entre o uso do copinho e as variáveis idade e escolaridade das mães, presença de gestação anterior, tempo de administração da dieta no copinho pelas mães e IG corrigida do bebê, corroborando o estudo que afirma que o uso do copinho não sofre interferência da IG do $R N{ }^{26}$. O resultado acima pode ser justificado pelo fato de RNs com 30 semanas de IG demonstram adequada habilidade para coordenar os movimentos de língua, para a deglutição e para respirar sem dificuldades enquanto se alimenta com o uso do copinho ${ }^{15}$.

O número e o volume do complemento também podem afetar o resultado da alimentação. Porém, não foi observada relação significativa no número de complementos ofertados aos bebês alimentados pelo copinho ${ }^{27}$, tal fato foi evidenciado no presente estudo.

As variáveis idade e escolaridade das mães e presença de gestação anterior são mencionadas como fatores críticos relacionados com a dificuldade inicial no estabelecimento da amamentação e desmame precoce, o mesmo não foi evidenciado nesse estudo quando relacionada ao uso do copinho ${ }^{28}$. Dessa forma, o uso do copinho é um método alter- nativo seguro para RNPTs que estão aprendendo a amamentar, fato demonstrado nesse estudo em que a técnica do copinho não depende das variáveis demonstradas acima para o seu manejo adequado ${ }^{29}$.

Em relação à orientação dos profissionais da saúde para o sucesso da administração da dieta no copinho pelas mães de RNPTs de baixo peso, o estudo apresentou relação estatisticamente significante $(p<0,05)$. Dado que corrobora as afirmações de que amamentar prematuros ainda é um desafio, mas é fato, desde que haja apoio e suporte apropriados, principalmente pelos profissionais da saúde.

São necessárias mais informações sobre a importância da amamentação para que as mães possam tomar decisões sobre a nutrição de seus filhos ${ }^{30}$, uma vez que as principais causas da não utilização de métodos alternativos de alimentação como o uso de copos e xícaras é a falta de orientação aos pais ${ }^{31}$.

No que se refere ao conhecimento das mães sobre a importância do uso do copinho, não foram observadas relações estatisticamente significantes $(R>0,05)$. As mães apenas conhecem o fato de que o uso da mamadeira pode apresentar problemas futuros ao bebê e que o uso do copo evita a 
confusão de bicos ${ }^{32}$. É interessante mencionar que nessa pesquisa, as respostas mais comuns apresentadas pelas mães quando questionadas sobre a importância do uso do copinho foi de que esta técnica evita o uso da mamadeira e faz com que o bebê seja alimentado no peito corretamente.

A posição do copo realizada pelas mães apresentou relação estatisticamente significante $(p<0,05)$, sendo importante para o sucesso da administração da dieta no copinho, visto que a posição inadequada deste pode promover alteração no ritmo de sorver o leite, pausas longas e escape prematuro de leite. O mesmo é evidenciado na literatura ao mencionar que a possível desvantagem do uso do copinho é em relação à sua posição, pelo risco de derramar o leite diretamente na boca do RN, podendo causar aspiração ${ }^{33,34}$.

Destaca-se também a importância da posição adequada do RN para o sucesso da administração da técnica do copinho, em que o bebê deve estar sentado ou semi-sentado no colo da mãe ${ }^{33}$. A postura do bebê apresentou relação estatisticamente significante $(p<0,05)$ no presente estudo, em que as mães posicionaram adequadamente o $\mathrm{RN}$ para a realização desta técnica. O mesmo não foi evidenciado com relação à postura da mãe $(p>0,05)$, em que a amostra apresentou-se homogênea, impossibilitando-nos de afirmar que nesse estudo a postura encontrava-se adequada.

O volume de leite ofertado na dieta do RN merece ser ressaltado, pois nesse estudo observou-se que quanto maior o volume maiores chances de ocorrerem pausas longas e escape prematuro de leite, relação esta estatisticamente significante $(p<0,05)$. O copo deve ser enchido $3 / 4$ da sua capacidade para que seja minimizado seu ângulo em relação ao lábio inferior do RN, mantendo o copo em uma posição que faça o leite disponível ao RN, para evitar que o leite seja derramado diretamente na sua boca ${ }^{20}$. Dessa forma, destaca-se a importância da variável volume do complemento durante a administração da dieta, visto que pode afetar o resultado da alimentação do bebê ${ }^{27}$.

Durante a avaliação da administração da dieta pela mãe utilizando o copinho, verificou-se baixo índice de acerto na forma de administrar a dieta no copinho, destacando o escape prematuro de leite $e$ pausas longas para sorver o leite, o que não condiz com a literatura que afirma que pausas longas para a retomada de ar durante a alimentação com o copinho parecem ser desnecessárias ${ }^{20}$. A justificativa mais plausível é de que durante a alimentação com o copinho, observa-se mais precisamente a falta de estimulação sensorial, ou o fato que os movimentos de sorver e lamber não são padrões no $\mathrm{RN}{ }^{35}$. A estimulação oral de RNPTs pode acelerar a aquisição da habilidade de sucção, facilitando a aceitação precoce de vários volumes de leite por via oral ${ }^{36}$.

Não foram observados episódios de tosse e/ou engasgos do RN durante a administração da dieta pelas mães, corroborando a literatura em que não foram descritas crises de apnéia ebroncoaspiração ${ }^{34}$. Apesar da técnica do copinho provocar espanto a todos aqueles que não estão familiarizados com ela, é constatada na rotina hospitalar a possibilidade do RN realizá-la, não sendo comum engasgos ou aspiração de leite pelos bebês que não possuem dificuldades de deglutição ${ }^{17}$.

\section{CONCLUSÃO}

A técnica do copinho é uma forma alternativa de transição da dieta do bebê, segura e fácil de ser administrada pelas mães, e este estudo veio a corroborar esses achados, pois independeu da idade $e$ escolaridade das genitoras, presença de gestação anterior, tempo de administração da dieta no copinho, conhecimento sobre a importância dessa técnica e idade gestacional corrigida da criança. Para otimizar os procedimentos realizados durante a técnica do copinho, o estudo demonstra a importância de orientações precisas dos profissionais da saúde sobre o uso do copo e volume de leite administrado, pois estas orientações foram fundamentais para 0 sucesso da administração adequada da dieta no copinho, principalmente no que se refere à posição do bebê. 


\section{ABSTRACT}

Purpose: to evaluate cup feeding manipulation by mothers of low weight preterm infants' and to analyze the aspects that interfere in the correct use of this technique. Methods: a cross-section correlation study was conducted. The sample included 30 pairs of mothers and babies. Data collection was based on patient' charts; interviews with the newborn' mothers and observing a diet offered in a cup feeding by mothers. The analysis of the results was based on the Software Statistical Package Social Sciences (SPSS) 13.0. We used descriptive statistical techniques through the distribution of the frequencies and the relatives percentages of each observed data. The Chi-square test and the Sperman Correlation Coeficient were used to analyze association between the variables $(p<0.05)$. Results: there is a significant relation between the babies' posture, the cup feeding, the volume given and the health professional guidance about cup feeding. Conclusion: mothers can manipulate the cup feeding, however, they need healthy professional guidance on this technique (cup feeding) and the milk's volume; mainly in relation to the care with the position of the glass and the volume of milk.

KEYWORDS: Milk, Human; Feeding Methods; Infant, Newborn; Infant, Premature

\section{REFERÊNCIAS}

1. Ciconi RCV, Venâncio SI, Escuder MML. Avaliação dos conhecimentos de equipe do programa da saúde da família sobre o manejo do aleitamento materno em um município da região metropolitana de São Paulo. Rev Bras Saúde Matern Infant. 2004; 4:193-202.

2. Pereira MJB, Reis MCG, Nakano MAS, Santos $\mathrm{CB}$, Villela MRG, Lourenço MCP. Breast feeding indicators in the municipality of Ribeirão Preto, São Paulo. Rev Bras Epidemiol. 2004; 7:36-43.

3. Furlan CEFB, Scochi CGS, Furtado MCC. Percepção dos pais sobre a vivência no método mãe canguru. Rev Latino-Am Enferm. 2003; 11:444-52.

4. Venâncio SI, Almeida H. Método mãe canguru: aplicação no Brasil, evidências científicas e impacto sobre o aleitamento materno. J Pediatr. 2004; 80:173-80.

5. Colameo AJ, Rea MF. O método mãe canguru em hospitais públicos do Estado de São Paulo, Brasil: uma análise do processo de implantação. Cad Saúde Pública. 2006; 22:597-607.

6. Andrade ISN, Guedes ZCF. Intervenção fonoaudiológica no processo de alimentação em bebês inseridos no método mãe-canguru. Fono Atual. 2005; 32:13-24.

7. Marques RFS, Lopez VFA, Braga JAP. O crescimento de crianças alimentadas com leite materno exclusivo nos primeiros 6 meses de vida. J Pediatr. 2004; 80:99-104.

8. Cormack BE, Bloomfield FH. Audit of feeding practices in babies $<1200 \mathrm{~g}$ or 30 weeks gestation during the first month of life. J Pediatr Child Health. 2006; 42(7-8):458-63.
9. Leite IG, Pinheiro AM, Brum LRG, Souza SBA. Relação da amamentação com o desenvolvimento do sistema estomatognático. J Bras Fonoaudiol. 2002; 3:237-42.

10. Sanches MT. Manejo clínico das disfunções orais na amamentação. J Pediatr. 2004; 80:155-62. 11. Nyqvist KH, Ewald U. Surface electromyography of facial muscles during natural and artificial feeding of infants: identification of differences between breast-, cup- and bottle- feeding. J Pediatr. 2006; 82(2):85-6.

12. Collins CT, Ryan P, Crowther CA, McPhee AJ, Paterson S, Hiller JE. Effect of bottles, cups, and dummies on breast feeding in preterm infants: a randomised controlled trial. BMJ. 2004; 329(7459):193-8. 13. Neiva FCB, Cattoni DM, Ramos JLA, Issler H. Desmame precoce: implicações para o desenvolvimento motor-oral. J Pediatr. 2003; 79:7-12.

14. Bühler KEB, Limongi SCO. O uso do copinho como método de alimentação de recém-nascidos pré-termo: revisão de literatura. Rev Soc Bras Fonoaudiol. 2004; 9:116-21.

15. Lang S, Lawrence CJ, Orme RL. Cup feeding: an alternative method of infant feeding. Arch Dis Child. 1994; 71(4):365-9.

16. Samuel $P$. Cup feeding: how and when to use it with term babies. Pract Midwife. 1998; 1(12):33-5.

17. Couto DE, Nemr K. Análise prática da técnica do copinho em hospitais amigos da criança nos estados do Rio de Janeiro e São Paulo. Rev CEFAC. 2005; 7(4):448-59.

18. Kuehl J. Cup feeding the newborn: what you should know. J Perinat Neonatal Nurs. 1997; 11(2):56-60.

19. Gupta A, Khanna K, Chattree S. Cup feeding: an alternative to bottle feeding in a neonatal intensive care unit. J Trop Pediatr. 1999; 45(2):108-10. 
20. Dowling DA, Meier PP, Difiore JM, Blatz M, Martin RJ. Cup-feeding for preterm infants: mechanics and safety. J Hum Lact. 2002; 18(1):13-20.

21. Howard CR, Howard FM, Lanphear B, Eberly S, Deblieck EA, Oakes D, Lawrence RA. Randomized clinical trial of pacifier use and bottle-feeding or cupfeeding and their effect on breastfeeding. Pediatrics. 2003; 111(3):511-8.

22. Gamburgo LJL, Munhoz SEM, Amstalden LG. Alimentação do recém-nascido: aleitamento natural, mamadeira e copinho. Fono Atual. 2002; 2:39-47.

23. Gomes CF, Trezza EM, Murade EC, Padovani CR. Surface electromyography of facial muscles during natural and artificial feeding of infants. J Pediatr. 2006; 82(2):103-9.

24. Ministério da Saúde. Sistema de Informação da Atenção Básica Indicadores 2001. Secretaria de Políticas de Saúde: Brasília; 2003.

25. Escobar AMU, Ogawa AR, Hilratsuka M, Kawashita MY, Teruya PY, Grisi S, Tomikawa SO. Aleitamento materno e condições sócio-econômico-culturais: fatores que levam ao desmame precoce. Rev Bras Saúde Mater Infant. 2002; 2:253-61.

26. Howard CR, Blieck EA, Hoopen CB, Howard FM, Lanphear BP, Lawrence RA. Physiologic stability of newborns during cup- and bottle-feeding. Pediatrics. 1999; 104(5 pt 2):1204-7.

27. Brown SJ, Alexander J, Thomas P. Feeding outcome in breast-fed term babies supplemented by cup or bottle. Midwifery. 1999; 15(2):92-6.

28. Sanches MTC. Amamentação: enfoque fonoaudiológico. In: Carvalho MR, Tamez RN. Amamenta- ção: bases científicas para prática profissional. Rio de Janeiro: Guanabara Koogan; 2002. p. 49-50.

29. Marinelli KA, Burke GS, Dodd VL. A comparison of the safety of cupfeedings in premature infants whose mothers intend to breastfeed. J Perinatol. 2001; 21(6):350-5.

30. Nascimento MB, Issler $\mathrm{H}$. Breastfeeding in premature infants: in-hospital clinical management. J Pediatr. 2004; 80(5 Suppl):S163-72.

31. Czernay APC, Bosco VL. A introdução precoce e o uso prolongado da mamadeira: ainda uma realidade. J Bras Odontopediatr. 2003; 6:138-44.

32. Cloherty M, Alexander J, Holloway I, Galvin K, Inch S. The cup-versus-bottle debate: a theme from an ethnographic study of the supplementation of breastfed infants in hospital in the United Kingdom. J Hum Lact. 2005; 21(2):151-62.

33. Thorley V. Cup feeding: problems created by incorrect use. J Hum Lact. 1997; 13(1):54-5.

34. Rocha NM, Martinez FE, Jorge SM. Cup or bottle for preterm infants: effects on oxygen saturation, weight gain, and breastfeeding. J Hum Lact. 2002; 18(2):132-8.

35. Alves MC, Araújo VC, Guedes ZCF. Habilidade na utilização dos utensílios copo e canudo por lactentes de 6 a 12 meses de idade. Fono Atual. 2005; 33:1-9.

36. Fucile S, Gisel E, Lau C. Oral stimulation accelerates the transition from tube to oral feeding in preterm infants. J Pediatr. 2002; 141(2):230-6.
RECEBIDO EM: 13/06/2007

ACEITO EM: 04/02/2008

Endereço para correspondência:

Av. Empresário Carlos da Silva Nogueira, 1256 ap. 404

Maceió - AL

CEP: $57036-540$

Tel: (82)32351224 / (82)88011112

E-mail: fonoaudiologiavivian@yahoo.com.br 